\title{
Solution for the electric potential distribution produced by sphere-plane electrodes using the method of images
}

(Solução para a distribuição do potencial elétrico produzido por eletrodos esfera-plano usando o método das imagens)

\author{
Fernando F. Dall'Agnol ${ }^{1}$ e Victor P. Mammana \\ Centro de Tecnologia da Informação Renato Archer, Rod. Dom Pedro I, Campinas, SP, Brasil \\ Recebido em 26/9/2008; Revisado em 11/3/2009; Aceito em 24/4/2009; Publicado em 23/9/2009
}

\begin{abstract}
The solution for the potential distribution between a spherical and planar electrode is briefly reviewed and derived in a simple way using the method of images. An analysis of the convergence of the potential, as a function of the number of image charges accounted, is also performed. Once the potential is obtained, the evaluation of the electric field, capacitance, energy of the system and the force between the electrodes is straightforward. The solution is illustrated with pictures, schematic representations and numerical examples.
\end{abstract}

Keywords: sphere-plane potential, electric potential, method of images, sphere-plane electrodes.

A solução para a distribuição do potencial em uma configuração de eletrodos esfera-plano é brevemente revisada e é obtida em detalhes usando-se o método das imagens. Uma vez que o potencial é obtido, o campo elétrico, a capacitância, a energia do sistema e a força entre os eletrodos podem ser deduzidos prontamente. É feita uma análise da convergência do potencial, como função do número de imagens consideradas no cálculo. A solução é ilustrada com figuras, representações esquemáticas e exemplos numéricos.

Palavras-chave: potencial esfera-plano, potencial elétrico, método das imagens, eletrodos esfera-plano.

\section{Introduction}

The solution of the potential distribution between a spherical and planar electrode constitutes an academic problem, which was already described by physicists almost 150 years ago. Nevertheless, it is not easy to find a reference with a solution that is immediately applicable. Although many authors, dealing with electrostatics, refer to the classical textbook by Smythe, unfortunately, this book does not present the potential distribution in a simple way [1].

The idea of image charge for field problems is due to Lord Kelvin, but Maxwell [2, Lodge [3, and Searle [?] extended the scope of the method. An excellent discussion on the distribution of the potential as a function of the system's coordinates was given by A. Foster in his $\mathrm{PhD}$ thesis 4 for a point charge between a sphere-plane capacitor. Foster uses the method of images to calculate the contribution of a point charge to the electric potential of the system.

Theoretical models for the electric potential distribution in the space between electrodes are useful for the calculation of the trajectories of charged particles and the prediction of flashover voltages over a given range of field conditions. Experiments based on high-voltage

\footnotetext{
${ }^{1}$ E-mail: fernando.dallagnol@cti.gov.br.

breakdown tests play an important rule in electrical engineering education. Real laboratory experiments designed to determine the voltage at breakdown have been combined with computer-based simulations and have produced stimulating teaching experiences. The experience gained with such a combination of teaching procedures is presented in the work of Lowther and Freeman [5]. The work of J.H. Cloete and J. van der Merwe 6] also describes an experiment for the determination of the voltage at breakdown by slowly decreasing the spacing between two conducting spherical electrodes. Their paper gives a detailed explanation on how to apply the method of images to model this practical problem. For many electrostatic systems, the method of images provides a simple solution, when solving Laplace equation would be very complicated, as is the case of the sphereplane system of electrodes.

In this paper we also use the method of images to obtain an analytical solution for the potential distribution in the space between a conducting sphere and a plane electrode. Our goals are to familiarize students with this method by solving the sphere-plane electrostatic problem and to present easy to use equations for the potential and the electric field. Once the potential distribution is obtained, the electric field distribution and 
system parameters like capacitance, stored energy and force between the electrodes can be deduced straightforwardly.

\section{Method of image charge}

Almost without exception, old and new textbooks on applied electromagnetism discuss the method of images, and this clearly shows the importance of the method for science and engineering. According to Binns, Lawrenson and Trowbridge [7, the essence of the method of images consists in replacing the effects of a boundary related to an applied field by distributions of charges "behind" the boundary line as illustrated in Fig. 1 for two configurations that will be combined to deduce the potential for sphere-plane electrodes. The first configuration consists of a point charge and a grounded conducting infinite plane and this is shown in Fig. 1(a). The second configuration consists of a point charge and a grounded conducting sphere shown in Fig. 1(b). The solid and dashed lines represent the real and virtual fields respectively. The point from where the dashed lines diverge is the image charge. The field pattern, and consequently, the potential distribution for the real electrodes system is equivalent as the one generated by two point charges. The advantage of the image method is that the evaluation of the potential with point charges is simple and straightforward.

A schematic representation of the sphere-plane electrode system is presented in Fig. 2. The two electrodes are subject to a potential difference $V$ and the deriva-

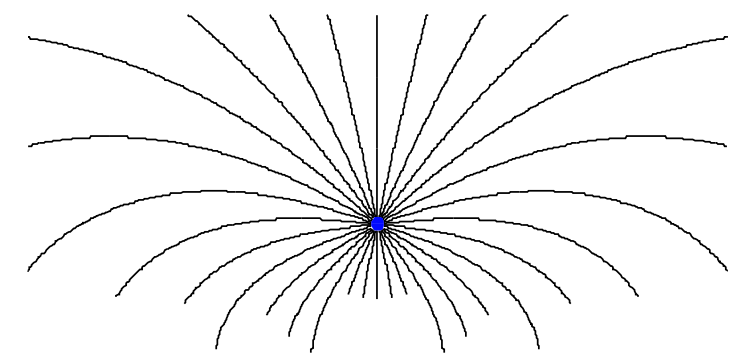

(a) tion is presented in cylindrical coordinates. According to the chosen reference frame, the conducting plane is placed at the coordinate $z=0$ and its electric potential $\phi_{p}$ is set to zero. The electric potential $\phi_{s}$ at the spherical electrode is $V$, being the potential difference between the spherical and the planar electrode. The sphere has radius $a$ and its center is placed at $z=z_{0}$. The minimum distance $d$ between the sphere and the grounded plane is $d=z_{0}-a$.

To find the potential for this system using image charges, we start considering an isolated sphere with charge $q_{0}$, which generates the potential $V$ at the surface. Then, $q_{0}$ can be expressed in terms of its resulting potential $V$ as

$$
q_{0}=\frac{a V}{k},
$$

where $k=9 \times 10^{9} \mathrm{Vm} / \mathrm{C}$ is the electrostatic constant. In the presence of the plane at $z=0$, charge $q_{0}$ generates an image of same magnitude and opposite sign $-q_{0}$ at position $-z_{0}$. The image charge $-q_{0}$ also generates an image in the sphere with position and magnitude given by

$$
\begin{gathered}
z_{1}=z_{0}-\frac{a^{2}}{2 z_{0}}, \\
q_{1}=\frac{a}{2 z_{0}} q_{0} .
\end{gathered}
$$

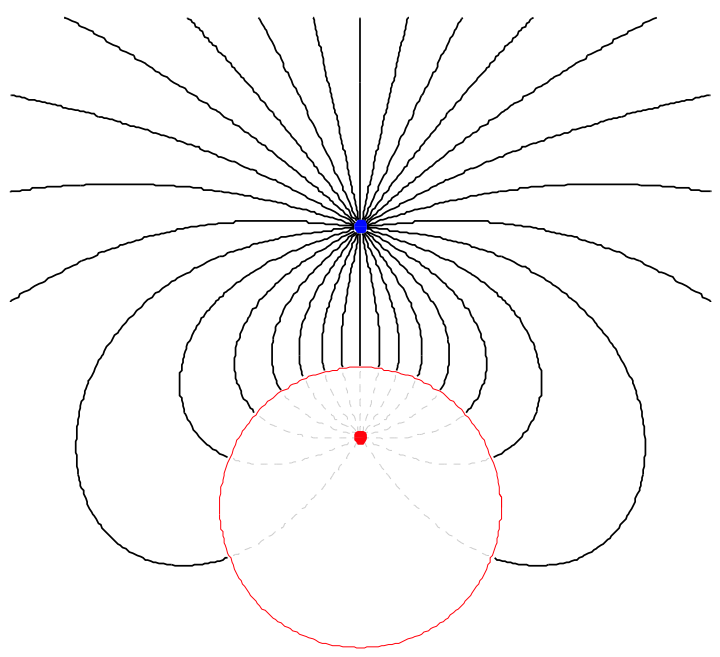

(b)

Figure 1 - The method of images for (a) a point charge and a grounded conducting plane and (b) a point charge and a grounded conducting sphere. The pattern of the electric field between point charge and electrode is identical to the pattern for two point charges. 


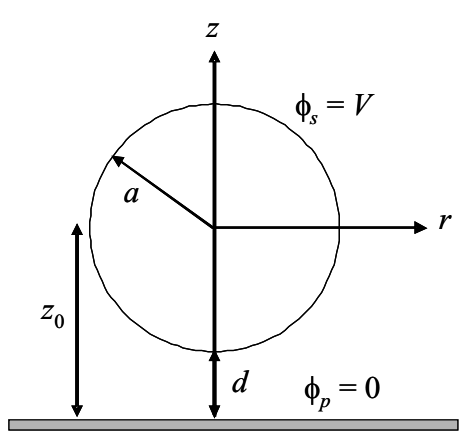

Figure 2 - Schematic representation of the sphere-plane electrodes in cylindrical coordinate system.

This situation is depicted in Fig. 3(a). The image charge $q_{1}$, in turn, generates $-q_{1}$ at the plane, which generates $q_{2}$ in the sphere and so on. Fig. 3(b) represents the final charge distribution. The position and magnitude of the $i^{t h}$ image charge are given by the recurrent relations

$$
\begin{gathered}
z_{i}=z_{0}-\frac{a^{2}}{z_{0}+z_{i-1}}, \\
q_{i}=\frac{a}{z_{0}+z_{i-1}} q_{i-1} .
\end{gathered}
$$

The derivation of Eqs. (2) to (5), which can be found in many textbooks, will not be repeated here.

It is convenient to define a normalized charge $\xi_{i}=q_{i} / q_{0}$, that will be used later. Dividing Eq. (5) by $q_{0}$ we get

$$
\xi_{i}=\frac{a}{z_{0}+z_{i-1}} \xi_{i-1}
$$

with $\xi_{0}=1$.

Charge distributions for three configurations of the problem are shown in Table 1 . The results show the effect of the minimum distance $d$ between the electrodes on the charge distribution in the spherical domain. The first six values of $z_{i}$ and $\xi_{i}$ for each $d$ are also presented. It can be noticed from this table that $\xi_{i} \rightarrow 0$ and $z_{i} \rightarrow z_{\infty}$ (constant) as $i \rightarrow \infty$. In these pictures the relative magnitude of the charges is represented in a gray scale from black, for $\xi_{0}$ (unity), to white, for $\xi_{\infty}$ (zero). In fact, these charges have images beneath the plane boundary, but they are not shown for the sake of simplicity. The position and magnitude of these image points are $-z_{i}$ and $-q_{i}$. The potential for the sphereplane is equivalent of the potential of these two groups of charges $\left\{q_{i},-q_{i}\right\}$.
Table 1 - Schematic illustrations for the image charges and their first 6 values of $\xi_{i}$ and $z_{i}$ for 3 distances and $a=1 \mathrm{~mm}$.

\begin{tabular}{lll}
\hline Image charges in the sphere & $\xi_{i}=q_{i} / q_{0}$ & $z_{i}(\mathrm{~mm})$ \\
\hline & $\xi_{0}=1$ & $z_{0}=1.01$ \\
& $\xi_{1}=0.495$ & $z_{1}=0.515$ \\
& $\xi_{2}=0.325$ & $z_{2}=0.354$ \\
& $\xi_{3}=0.238$ & $z_{3}=0.277$ \\
& $\xi_{4}=0.185$ & $z_{4}=0.233$ \\
& $\xi_{5}=0.149$ & $z_{5}=0.206$ \\
& $\xi_{6}=0.122$ & $z_{6}=0.187$ \\
& & $z_{\infty}=0.142$ \\
\hline & $\xi_{0}=1$ & $z_{0}=1.1$ \\
& $\xi_{1}=0.455$ & $z_{1}=0.645$ \\
& $\xi_{2}=0.260$ & $z_{2}=0.527$ \\
& $\xi_{3}=0.160$ & $z_{3}=0.485$ \\
& $\xi_{4}=0.101$ & $z_{4}=0.469$ \\
& $\xi_{5}=0.064$ & $z_{5}=0.463$ \\
& $\xi_{6}=0.041$ & $z_{6}=0.460$ \\
& & $z_{\infty}=0.458$ \\
\hline & $\xi_{0}=1$ & $z_{0}=2.0$ \\
& $\xi_{1}=0.25$ & $z_{1}=1.750$ \\
& $\xi_{2}=0.0667$ & $z_{2}=1.733$ \\
& $\xi_{3}=0.0178$ & $z_{3}=1.732$ \\
& $\xi_{4}=0.0047$ & $z_{4}=1.732$ \\
& $\xi_{5}=0.0013$ & $z_{5}=1.732$ \\
& $\xi_{6}=0.0003$ & $z_{6}=1.732$ \\
& & \\
\hline
\end{tabular}

\section{Solution for the potential, the elec- tric field and other physical quanti- ties}

The potential due to a charge of index $i$ in the sphere and its image in the plane is given by

$\phi_{i}=k\left(\frac{q_{i}}{\left[\left(z-z_{i}\right)^{2}+r^{2}\right]^{1 / 2}}-\frac{q_{i}}{\left[\left(z+z_{i}\right)^{2}+r^{2}\right]^{1 / 2}}\right)$.

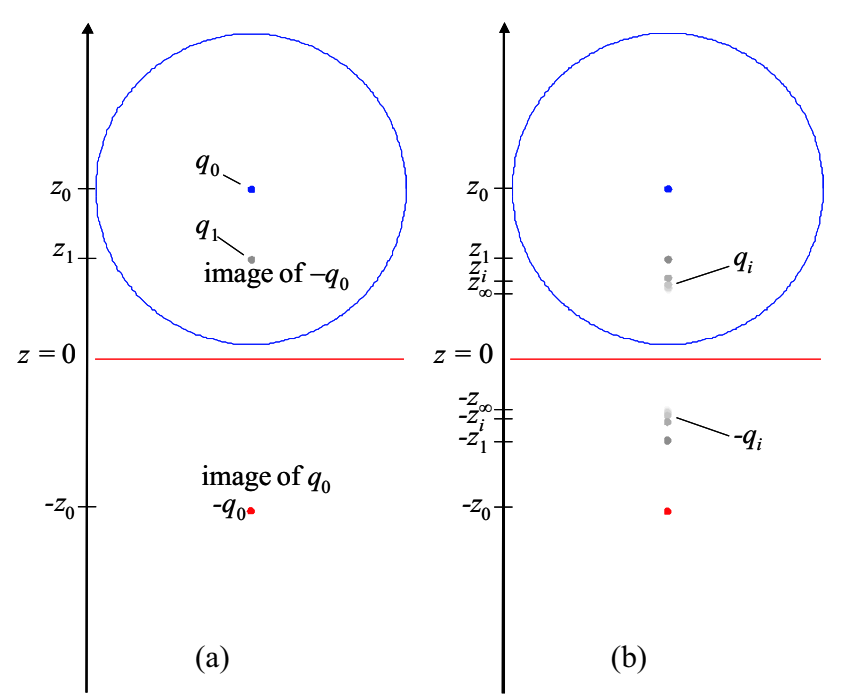

Figure 3 - Composition of the two systems of image points. (a) Representation of the first two images generated by the real charge and (b) the final distribution of image charges. 
The potential due to all charges is completely determined by summing $\phi_{i}$ and using Eqs. (1) and (5) in Eq. (7), results in

$$
\phi(r, z)=a V \sum_{i=0}^{\infty} \frac{\xi_{i}}{\left[\left(z-z_{i}\right)^{2}+r^{2}\right]^{1 / 2}}-\frac{\xi_{i}}{\left[\left(z+z_{i}\right)^{2}+r^{2}\right]^{1 / 2}}
$$

It is worth to point out that Eq. (8) is a solution for point charges, which is not the actual system of a sphereplane. The solution in Eq. (8) is equivalent to the solution of the sphere-plane system only for $\left(z-z_{0}\right)^{2}+r^{2} \geq a^{2}$ (outside the sphere) and for $z \geq 0$ (above the plane). For the region inside the sphere $\phi=V$ at any point and beneath the plane $\phi=0$ at any point. To account for these regions Eq. (8) has to be redefined as

$$
\phi(r, z)=\left\{\begin{array}{cc}
0 & z \leq 0 \\
V & r^{2}+\left(z-z_{0}\right)^{2} \leq a^{2} \\
a V \sum_{i=0}^{\infty} \frac{\xi_{i}}{\left[\left(z-z_{i}\right)^{2}+r^{2}\right]^{1 / 2}}-\frac{\xi_{i}}{\left[\left(z+z_{i}\right)^{2}+r^{2}\right]^{1 / 2}} & \text { otherwise }
\end{array}\right.
$$

Once $\phi$ is given, electric field, capacitance, electrostatic energy and force on the electrodes can be obtained promptly. The electric field can be obtained from $\mathbf{E}(r, z)=-\nabla \phi$ and can be written as

$$
\begin{aligned}
& E_{r}(r, z)=a V r \sum_{i=0}^{\infty} \frac{\xi_{i}}{\left[\left(z-z_{i}\right)^{2}+r^{2}\right]^{3 / 2}}-\frac{\xi_{i}}{\left[\left(z+z_{i}\right)^{2}+r^{2}\right]^{3 / 2}}, \\
& E_{z}(r, z)=a V \sum_{i=0}^{\infty} \frac{\xi_{i}\left(z-z_{i}\right)}{\left[\left(z-z_{i}\right)^{2}+r^{2}\right]^{3 / 2}}-\frac{\xi_{i}\left(z+z_{i}\right)}{\left[\left(z+z_{i}\right)^{2}+r^{2}\right]^{3 / 2}},
\end{aligned}
$$

where $E_{r}$ and $E_{z}$ are the components of the field in $r$ and $z$ directions respectively, so that $\mathbf{E}(r, z)=E_{r} \hat{\mathbf{r}}+E_{z} \hat{\mathbf{z}}$. Fig. 4 shows the potential distribution as a density plot and the electric field lines. Details on the procedure to obtain these figures are shown in Appendix B.

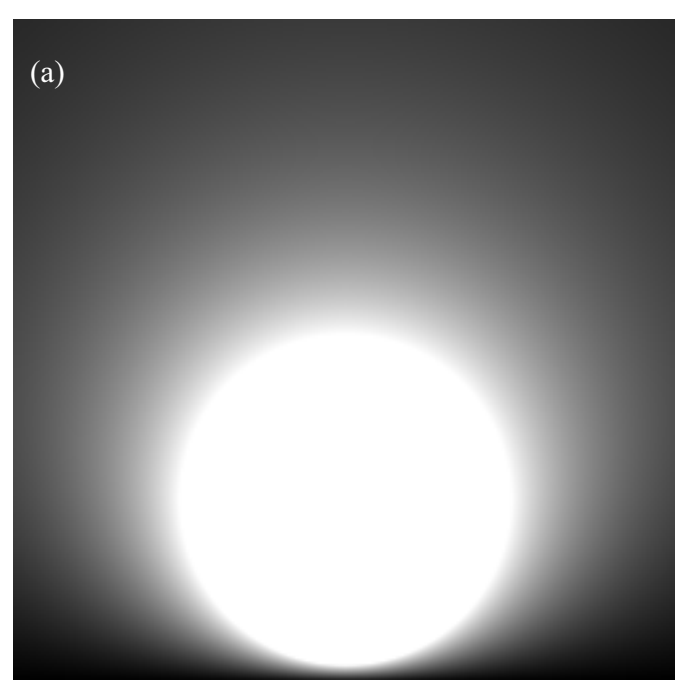

(b)

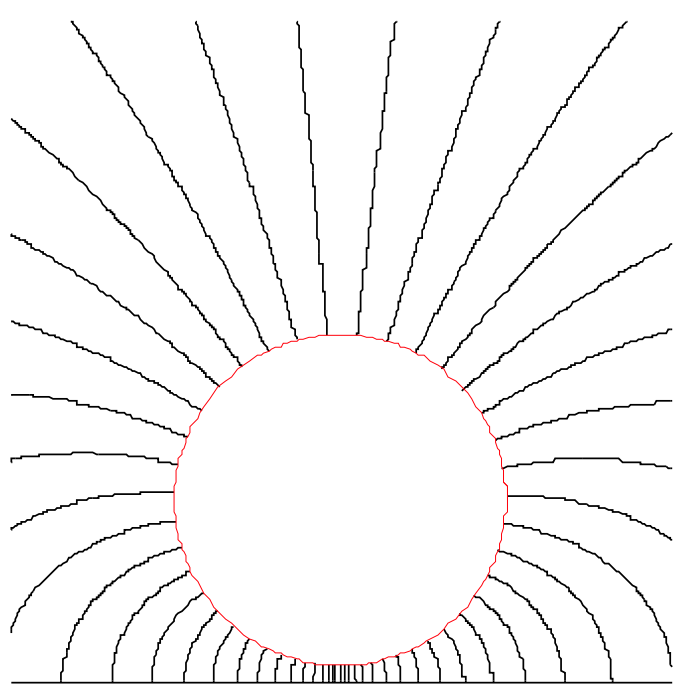

Figure 4 - (a) Density plot of the potential distribution from white, for $\phi=V$, to black, for $\phi=0$; and (b) the field lines between the electrodes, where the field strength is represented by the density of the lines. 
The sphere-plane electrode system is a capacitor of which the total charge $q$ is the sum of all charges in the sphere, given by

$$
q=\sum_{i=0}^{\infty} q_{i}=q_{0} \sum_{i=0}^{\infty} \xi_{i} .
$$

From the sequences of $\xi_{i}$ in Table 1 it can be seen that the larger $d$ is the faster $\xi_{i}$ converges. So the number of terms to be summed for $q$ in Eq. (12), to reach a specified accuracy, depends on $d$. A convergence analysis of $q$ and of $\phi$ is presented in the next section.

The capacitance is obtained from $C=q / V$. Combining Eqs. (1) and (12) results in

$$
C=\frac{a}{k} \sum_{i=0}^{\infty} \xi_{i} .
$$

The electrostatic energy $U=1 / 2 C V^{2}$ is given by

$$
U=\frac{a V^{2}}{2 k} \sum_{i=0}^{\infty} \xi_{i}
$$

and the force between sphere and plane $F=-\partial U / \partial z_{0}$ is

$$
F=-\frac{a V^{2}}{2 k} \sum_{i=0}^{\infty} \xi_{i}^{\prime}
$$

The prime mark indicates a differentiation with respect to $z_{0}$. In this way, differentiation of (6) leads to the following recurrent relation to compute $\xi_{i}^{\prime}$

$$
\xi_{i}^{\prime}=\frac{a}{z_{0}+z_{i-1}}\left[-\xi_{i-1} \frac{1+z_{i-1}^{\prime}}{z_{0}+z_{i-1}}+\xi_{i-1}^{\prime}\right],
$$

where $z_{i}^{\prime}$ is given by

$$
z_{i}^{\prime}=1+a^{2} \frac{z_{i-1}^{\prime}}{\left(z_{0}+z_{i-1}\right)^{2}} .
$$

The starting conditions for the recurrent relations above are $\xi_{0}^{\prime}=0$ and $z_{0}^{\prime}=1$. All formulas are summarized in Appendix A. The treatment given above can also be applied to two spheres. If the spheres have the same size the formulas for the potential distribution are identical to Eq. (8). In this case the symmetry plane of the sphere-sphere problem becomes the plane of the sphere-plane system.

\section{Convergence analysis of charge and potential}

The number of terms needed for the total charge $q$ in Eq. (12) to converge depends only on the ratio $z_{0} / a$. Table 2 shows the number of terms needed for $q$ to converge to $99.9 \%$ of its asymptotic value as a function of $z_{0} / a$.

Table 2 - Number of terms needed for the product $k . q$ to converge as a function of $z_{0} / a$.

\begin{tabular}{lcc}
\hline$z_{0} / a$ & $k . q\left(\times 10^{-3} \mathrm{Vm}\right)$ & $\begin{array}{c}\text { Number of terms } \\
\text { to converge to } 99.9 \%\end{array}$ \\
\hline 2 & 1.34 & 5 \\
1.1 & 2.15 & 13 \\
1.01 & 3.23 & 34 \\
1.001 & 4.36 & 75 \\
\hline
\end{tabular}

An analysis of the convergence of $\phi$ may be important as, for example, in fitting processes, where fitting parameters must be varied, then evaluated, and compared to experimental results repeatedly. A fitting procedure may take a lot of time and one must avoid computing unnecessary terms of $\phi$. This analysis aims to give a good insight about the convergence behavior, i.e. where the convergence is faster/lower, how the convergence varies with $z_{0} / a$. In Fig. 5 we present the convergence behavior for two cases of $z_{0} / a$. In this figure the numbers represent the number of terms needed for $\phi$ in Eq. (8) to converge to $99.9 \%$ of the asymptotic value at that point of the space. It can be seen that the region under the sphere needs more terms to converge. The number of terms needed is, at most, up to 50 even for $z_{0} / a$ as small as 1.01. For much different cases than the examples used here, like: much higher accuracy, much wider range of the coordinates, ratio $z_{0} / a$ much closer to unity, etc, requires an analysis for these specific cases. The asymptotic values of $q$ and $\phi$ are taken with 100 terms in their sum. 

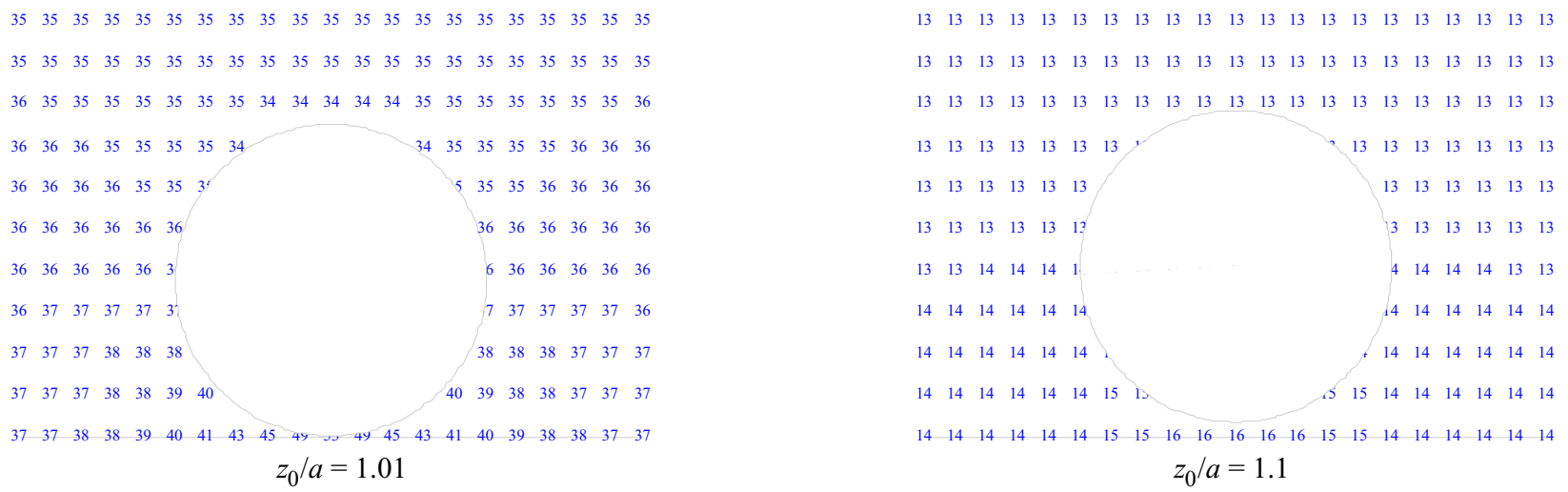

Figure 5 - Numbers indicate how many terms are necessary for the potential to converge to $99.9 \%$ of its asymptotic value at that point of the space.

\section{Conclusion}

We developed the solution for the potential, electric field, capacitance, energy and force for electrodes in a sphere-plane configuration using the method of image charges. Our equation, represented in a cylindrical coordinate system, can easily and conveniently be applied to systems with plane-sphere geometry. The methodology for solving this problem may be used in several other problems of electrostatics. We are confident that our treatment can conveniently be applied by students and engineers working on electrostatics.

\section{Acknowledgments}

We greatly acknowledge the help of Dr. Pablo Paredez, Ms. Aline Marque and Dr. Daniel den Engelsen for the help in preparing this manuscript. Authors are grateful to the Brazilian funding agencies CNPq and Fapesp for financial support.

\section{Appendix A: Formulary}

\begin{tabular}{|c|c|}
\hline Image charge position & $z_{i}=z_{0}-\frac{a^{2}}{z_{0}+z_{i-1}}$ for $i>0$ \\
\hline Initial (central) charge & $q_{0}=\frac{a V}{k}$ \\
\hline Image charge magnitude & $q_{i}=\frac{a}{z_{0}+z_{i-1}} q_{i-1}$ for $i>0$ \\
\hline Relative charge magnitude $q_{i} / q_{0}$ & $\xi_{i}=\frac{a}{z_{0}+z_{i-1}} \xi_{i-1}$ for $i>0$ \\
\hline$\partial z_{i} / \partial z_{0}$ & $\begin{array}{l}z_{0}^{\prime}=1 \\
z_{i}^{\prime}=1+a^{2} \frac{z_{i-1}^{\prime}}{\left(z_{0}+z_{i-1}\right)^{2}} \text { for } i>0\end{array}$ \\
\hline$\partial \xi_{i} / \partial z_{0}$ & $\begin{aligned} \xi_{0}^{\prime} & =0 \\
\xi_{i}^{\prime} & =\frac{a}{z_{0}+z_{i-1}}\left[-\xi_{i-1} \frac{1+z_{i-1}^{\prime}}{z_{0}+z_{i-1}}+\xi_{i-1}^{\prime}\right] \text { for } i>0\end{aligned}$ \\
\hline Total charge & $q=q_{0} \sum_{i=0}^{\infty} \xi_{i}$ \\
\hline Potential & $\phi(r, z)=\left\{\begin{array}{cc}0 & z \leq 0 \\
V & r^{2}+\left(z-z_{0}\right)^{2} \leq a^{2} \\
a V \sum_{i=0}^{\infty} \frac{\xi_{i}}{\left[\left(z-z_{i}\right)^{2}+r^{2}\right]^{1 / 2}}-\frac{\xi_{i}}{\left[\left(z+z_{i}\right)^{2}+r^{2}\right]^{1 / 2}} & \text { otherwise }\end{array}\right.$ \\
\hline Electric field: $r$ component & $E_{r}(r, z)=a V r \sum_{i=0}^{\infty} \frac{\xi_{i}}{\left[\left(z-z_{i}\right)^{2}+r^{2}\right]^{3 / 2}}-\frac{\xi_{i}}{\left[\left(z+z_{i}\right)^{2}+r^{2}\right]^{3 / 2}}$ \\
\hline Electric field: $z$ component & $E_{z}(r, z)=a V \sum_{i=0}^{\infty} \frac{\xi_{i}\left(z-z_{i}\right)}{\left[\left(z-z_{i}\right)^{2}+r^{2}\right]^{3 / 2}}-\frac{\xi_{i}\left(z+z_{i}\right)}{\left[\left(z+z_{i}\right)^{2}+r^{2}\right]^{3 / 2}}$ \\
\hline Capacitance & $C=\frac{a}{k} \sum_{i=0}^{\infty} \xi_{i}$ \\
\hline Energy & $U=\frac{a V^{2}}{2 k} \sum_{i=0}^{\infty} \xi_{i}$ \\
\hline Force & $F=-\frac{a V^{2}}{2 k} \sum_{i=0}^{\infty} \xi_{i}^{\prime}$ \\
\hline
\end{tabular}




\section{Appendix B: Algorithms and programs for rendering graphics}

Once the equations for the potential and the electric fields are established their corresponding graphics can be rendered for a visual presentation of the solution. This is not a trivial procedure. Many high level program languages are available for algebraic manipulation and graphics. In this section we will line out a procedure in Mathematicaß] [8]- a software with a very high level programming language- that we used to render the figures shown in this article. Next sections will provide an overall procedure to do the programs in Mathematica and will present the programs used.

\section{B1. Image charges in the sphere}

The figures shown in Fig. 3 and Table 1 was obtained as follows:

1. Numerical values are attributed to $z_{0}, a$.

2. Normalized charges $\xi_{i}$ and their positions $z_{i}$ are calculated for $i$ from 0 to 100, which is more than sufficient.

3. The representation of the sphere, the plane and the points $\left(0, z_{i}\right)$ are drawn using built-in commands Circle[...], Line[...] and Point[...] respectively. The points are rendered in gray level using command GrayLevel[...].

4. Graphics are merged and rendered.

The program used is shown below. An animation can be found in the internet with URL given in Ref. [9].

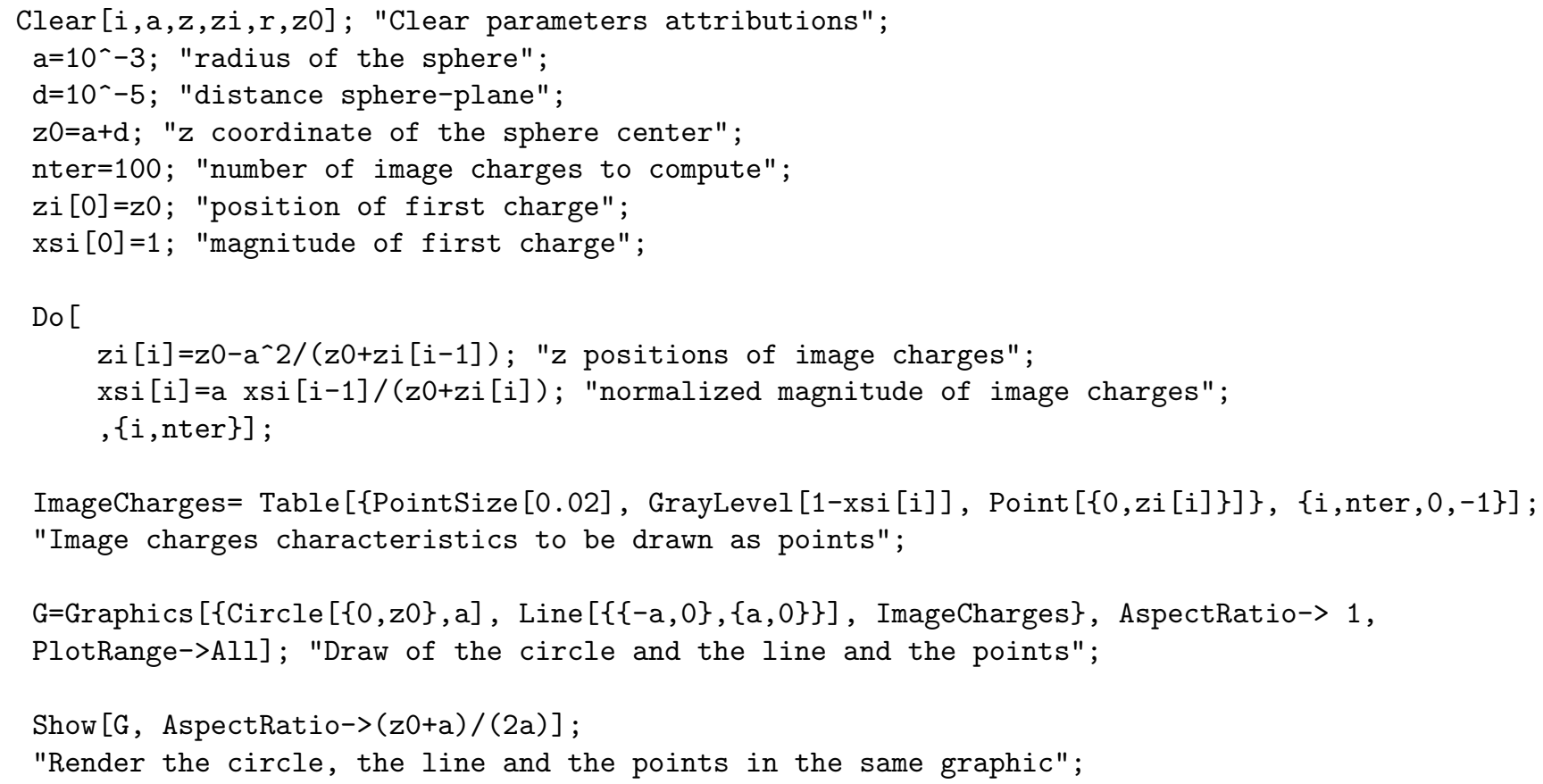

\section{B2. Density plot of the potential}

To render Fig. 4(a):

1. We attributed numbers to parameters $a$ and $z_{0}$.

2. We computed $z_{i}$ and $\xi_{i}$ for $i$ from 0 to 100.

3. We wrote the potential as in Eq. (9) like $\phi=\operatorname{If}\left[\right.$ condition, then, else], where condition is $\left(z-z_{0}\right)^{2}+r^{2} \geq a^{2}$, block then must be the expression for $\phi$ outside the sphere and block else is the expression for $\phi$ inside the sphere $(V)$.

4. We rendered the potential with built-in command DensityPlot $[\phi(r, z)]$.

The program used is shown below. See also URL in Ref. [10].

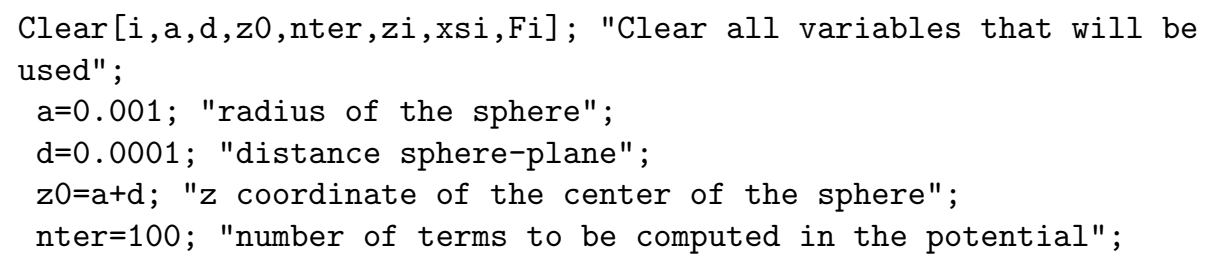




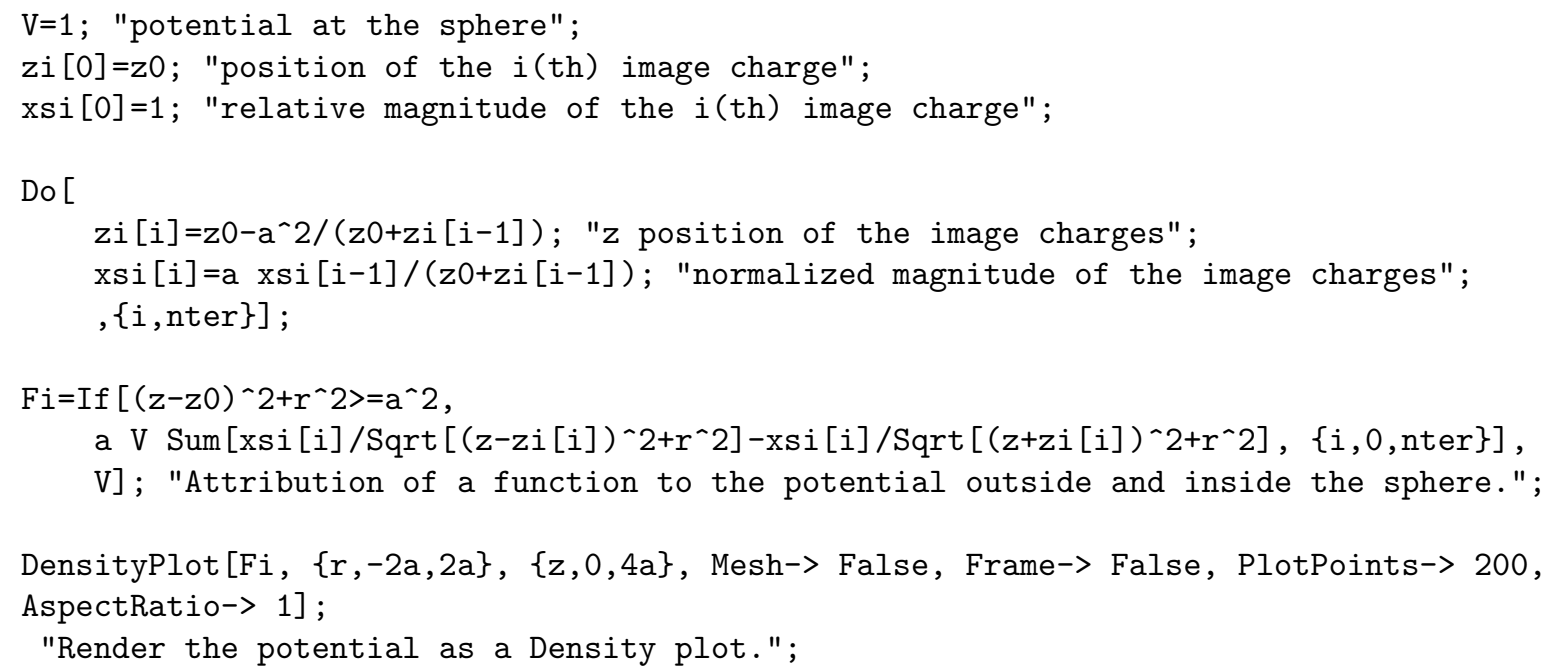

\section{B3. Field lines between the electrodes}

To render Fig. 4(b):

1. We attributed numerical values to $a, z_{0}$ and few other parameters used.

2. We computed $z_{i}$ and $\xi_{i}$ for $i$ from 0 to 100 .

3. We attributed expressions for the electric field functions $E_{r}$ and $E_{z}$ as in Eqs. (10) and (11).

4. Points on the circle that represents the sphere where selected as starting points of the electric field line. The starting point of each electric field line is not equally spaced from each other. Their spacing is proportional to the strength of the electric field, relative to the maximum field at the bottom of the sphere. This is done so the reader can visualize the field strength by the density of the field lines.

5. The field line is built, point by point, using the coordinate of the previous point plus a constant step toward the field direction to calculate the next point of the field line. New points are calculated until the field line reaches the border of the region to be shown in the graphic. The points are stored in a list and plotted.

The program is shown below. See also Ref. [11. Fig. 1(a) and (b) are particular cases of this program and will not be shown here. Animations of Fig. 1(a) can be seen in Ref. [12.

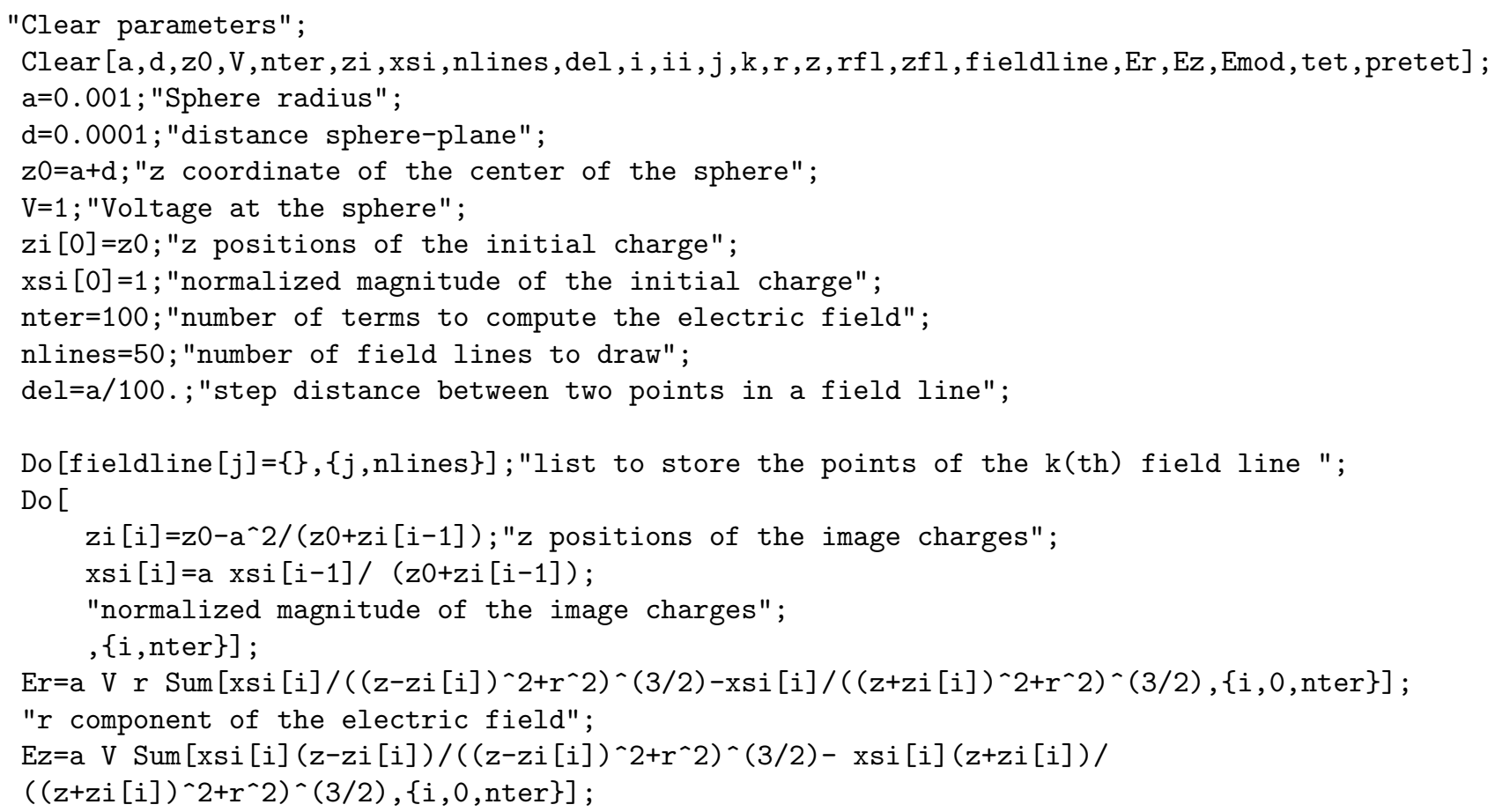




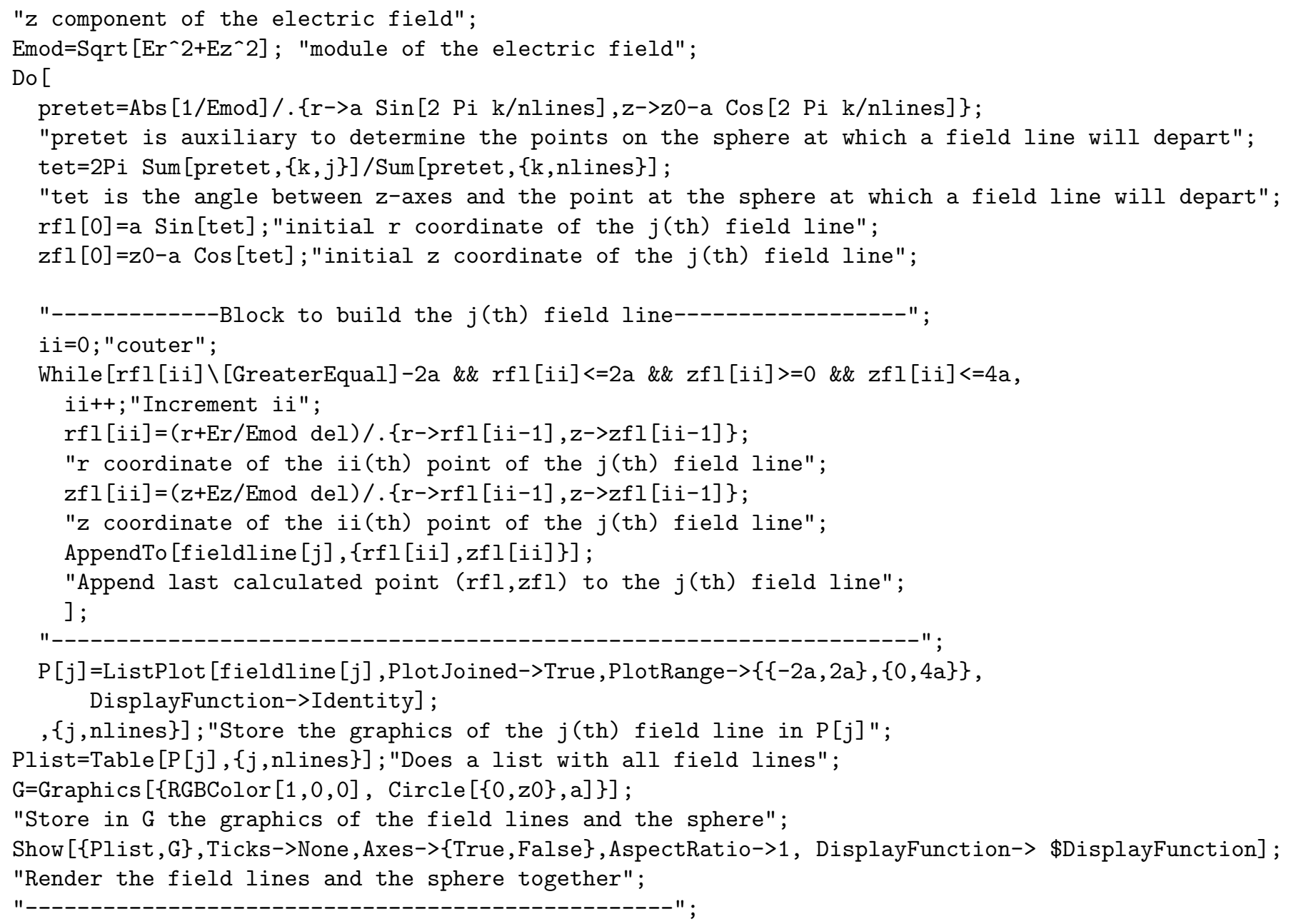

\section{References}

[1] W.R. Smythe, Static and Dynamic Electricity (McGraw-Hill, New York, 1950), 2nd ed.

[2] J.C. Maxwell, A Treatise in Electricity and Magnetism (Dover, New York, 1958), v. 1 3rd ed.

[3] O.J. Lodge, Proc. Phys. Soc. London. 2, 24 (1875).

[4] A. Foster, Theoretical Modeling of Non-contact Atomic Force Microscopy on Insulators. PhD Thesis, University College London, 2000. Available at http://www.fyslab.hut.fi/ asf/physics/thesis1/ thesis1.html, in 01/09/2009.

[5] D.A. Lowther and E.M. Freeman, IEEE Trans. Educ. 36, 219 (1993).

[6] J.H. Cloete and J. van der Merwe, IEEE Trans. Educ. 41, 141 (1998).
[7] K.J. Binns, P.J. Lawrenson and C.W. Trowbridge, The Analytical and Numerical Solution of Electric and Magnetic Fields (John Wiley \& Sons, Chichester, 1992), p. 93.

[8] Available at http://en.wikipedia.org/wiki/ Mathematica_(programming_language)

[9] Available at http://www.youtube.com/watch?v= mnTXosm9VRY See also http://www.youtube.com/ watch?v=DBSjvGSE8tk, in 02/10/2009.

[10] Available at http://www.youtube.com/watch?v= rpBDLtzt_VI, in 02/10/2009.

[11] Available at http://www.youtube.com/watch?v= ZHVpaqPVuBU, in 02/10/2009.

[12] Available at http://www.youtube.com/watch?v= HmZjHGG_4cQ, in 02/10/2009. 